\title{
Potentialities of slow and fast light in optical fibers
}

\author{
Luc Thévenaz*a , Miguel Gonzalez Herraez ${ }^{\mathrm{b}}$, Sang-Hoon Chin ${ }^{\mathrm{a}}$ \\ ${ }^{a}$ Institute of Electrical Engineering, École Polytechnique Fédérale de Lausanne, \\ STI-GR-SCI Station 11, 1015 Lausanne, Switzerland; \\ ${ }^{\mathrm{b}}$ Dept. of Electronics, University of Alcalá, Alcalá de Henares 28805, Spain.
}

\begin{abstract}
The ubiquitous role of optical fibers in modern photonic systems has stimulated research to realize slow and fast light devices directly in this close-to-perfect transmission line. Recent progress in developing optically-controlled delays in optical fibers, operating under normal environmental conditions and at telecommunications wavelengths, has paved the way towards real applications for slow and fast light. Advanced schemes can be realized thanks to the extremely flexible possibility to shape the gain spectrum to make it optimized for applications. Ultra wide bandwidth, delaying with flat amplitude response and lower distortion were successfully demonstrated this way.
\end{abstract}

Keywords: Fiber optics, Nonlinear optics, Slow \& fast light, stimulated Brillouin scattering.

\section{INTRODUCTION}

Given the limited possibilities to engineer the dispersion curve for standard singlemode fibers without creating complex photonic crystal structures, much research on fiber-based delays has been directed towards solutions based on spectral resonances to generate slow and fast light ${ }^{1}$.

Among all parametric processes observed in optical fibers stimulated Brillouin scattering (SBS) turns out to offer the best amplification per unit pump power. This high efficiency is moreover coupled to an intrinsic narrowband nature of the interaction, which makes it particularly suitable to make efficient delay lines based on slow light. It is therefore not surprising that the first demonstrations of slow and fast light in optical fibers were realized using the SBS interaction ${ }^{2,3}$, leading to impressive results such as very long delays ${ }^{4}$ and substantial index changes to achieve extreme propagation conditions such as superluminal and negative velocities 5 .

However, despite these impressive achievements obtained using simple benchtop set-ups, it was soon identified that the narrowband nature of stimulated Brillouin scattering makes it unsuitable for broadband data communications, since the $30 \mathrm{MHz}$ linewidth of the gain spectral distribution restricts the full delaying effect to pulses longer than $40 \mathrm{~ns}$. A simple solution has now been discovered to overcome the very narrowband nature of this interaction. This solution is based on the unique property that Brillouin spectral resonances produced by different laser lines can be combined and superposed to result in an enlarged spectral distribution. The laser lines can be generated by distinct lasers or as the result of a simple modulation. This unique feature of SBS, which will be described in more detail in the following section, overcomes the bandwidth hurdle which was blocking the use of SBS in real applications and has given rise to numerous reports of advanced schemes realizing functions that would be very difficult to achieve using other interactions.

This spectral tailoring capability in SBS has also been used to manage the distortion of the pulses travelling in the slow light system ${ }^{6}$. The idea is to optimize the pump spectrum so as to minimize the distortion in the signal pulse. This possibility was thoroughly explored by independent teams ${ }^{7-9}$, even finding an "optimum" gain and loss profile to yield the best performance in terms of delay and distortion. This has stimulated and unfortunately misled some teams to make a further step by proposing to compensate the amplitude broadening by the phase broadening and even by the fiber group velocity dispersion ${ }^{10,11}$. In the final section of this paper we shall discuss some fundamental limitations related to pulse broadening and demonstrate that the broadening obtained in the optimum gain profile cannot be compensated by a simple linear system.

*luc.thevenaz@epfl.ch; phone +41 21693 4774; http://people.epfl.ch/luc.thevenaz

Advances in Slow and Fast Light II, edited by Selim M. Shahriar, Philip R. Hemmer, John R. Lowell, Proc. of SPIE Vol. 7226, 72260C · C 2009 SPIE · CCC code: 0277-786X/09/\$18 · doi: 10.1117/12.816337 


\section{GAIN SPECTRAL SHAPING AND PERFORMANCE OPTIMIZATION USING PUMP MODULATION}

The linear dependence of the SBS gain on pump power has a crucial impact on the design of delaying schemes - if the pump power spectrum is polychromatic it can be decomposed into a sum of monochromatic waves. Each of these waves produces its own SBS spectral resonances and thus delaying effects for the fraction of the signal spectrum that matches its narrowband resonance. The overall effect on the signal will be the summation of all these separate and partial contributions. This situation can be represented by a fictitious monochromatic pump stimulating a fiber medium showing an effective Brillouin gain spectral distribution $g_{B}^{e f f}(v)$. The gain spectral distribution corresponds to the convolution of the natural narrowband Brillouin gain spectrum $g_{B}(v)$ with the normalized pump power spectrum

$$
g_{B}^{e f f}(v)=g_{B}(v) \otimes \frac{P_{\text {pump }}(v)}{P_{\text {pump }}^{\text {tot }}} \quad \text { with } \quad P_{\text {punp }}^{\text {tot }}=\int_{0}^{\infty} P_{\text {punn }}(v) d v
$$

where $\otimes$ conventionally denotes the convolution operator, $P_{\text {pump }}(v)$ the pump power spectral density in $\mathrm{W} / \mathrm{Hz}$ and $P_{\text {punp }}^{\text {tot }}$ the total pump power in $\mathrm{W}$.

The relationship between effective Brillouin gain spectral distribution and pump power spectral density has a tremendous impact on applications for signal transformation through SBS. It means that the gain spectral distribution can be drastically modified and shaped by the pump power spectrum. This is depicted in Fig. 1, showing that the result of the convolution is to smooth the actual pump power spectrum. Actually the spectral distribution of the effective Brillouin gain $g_{B}^{e f f}$ is essentially given by the pump power spectrum when it is much broader than the natural Brillouin resonance $(\sim 30 \mathrm{MHz}$ at $\lambda=1550 \mathrm{~nm})$.
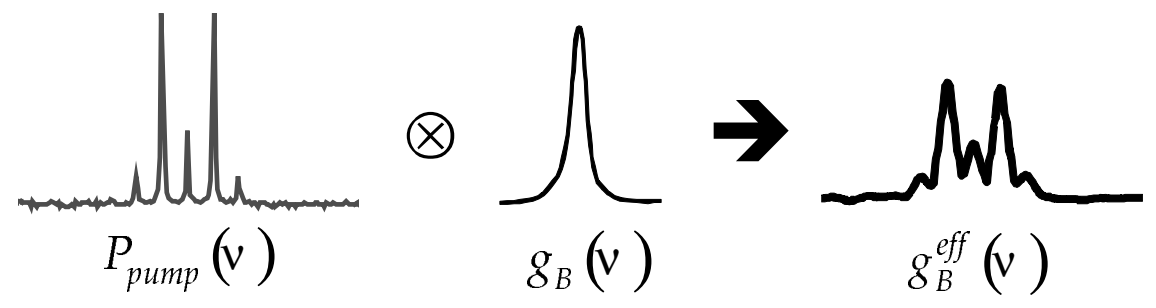

Fig. 1. The effective Brillouin gain spectrum $g_{B}^{\text {eff }}$ is given by the convolution of the pump power spectral distribution $P_{\text {pump }}(v)$ and the intrinsic Brillouin gain spectrum $g_{B}$, offering the possibility to synthesize tailored gain spectral distribution.

\subsection{Modulation using a discrete-line spectrum}

The ability to modify the SBS gain spectrum was soon identified after the pioneering experiments on SBS delaying and the first demonstration was realized using the simplest polychromatic spectrum made of just two frequency components ${ }^{12}$. The result of the convolution is to produce a double resonance and if the peak separation is comparable to the natural Brillouin linewidth, the overlapping of the resonances generates a reverse slope and fast light can be realized in gain regime 6 . This situation is depicted in Fig. 2b, showing the additional advantage that the delaying effect is produced with a much reduced signal amplification. It turns out that this latter feature has a limited advantage, since a large gain is still present in the centre of the two resonance peaks that may amplify the spontaneous emission and limit the maximum pump power for generating the delays. This cannot be solved by producing slow light in the loss regime ${ }^{13}$ since gain resonance peaks will be generated as well symetrically in the Stokes band.

But this demonstration clearly shows that these new schemes of pump modulation can be used to slow light. In that particular case of the two-frequency pump, the delay can be continuously tuned by changing the frequency separation between the two spectral lines instead of varying the pump power, even allowing the ability to switch from slow light to fast light propagation ${ }^{12}$. 
Modifying the actual spectral shape of the resonance is also interesting for the optimization of the delay effect. For instance an index slope steeper than observed for a natural Lorentzian distribution can be produced within the same bandwidth by a properly shaped gain spectrum. In addition, this reshaping can also significantly reduce the distortion experienced by the signal. Through this approach, interesting studies were carried out by varying the relative amplitude and the frequency separation between evenly spaced frequency components and the best slow light response was obtained for a three-tone pump spectrum with identical amplitudes for each line ${ }^{14,15}$, as depicted in Fig. 2c. The scheme can be further extended up to 11 lines of equal amplitudes using a combination of phase and amplitude modulation ${ }^{16,17}$ to eventually get a broadband flat spectrum.

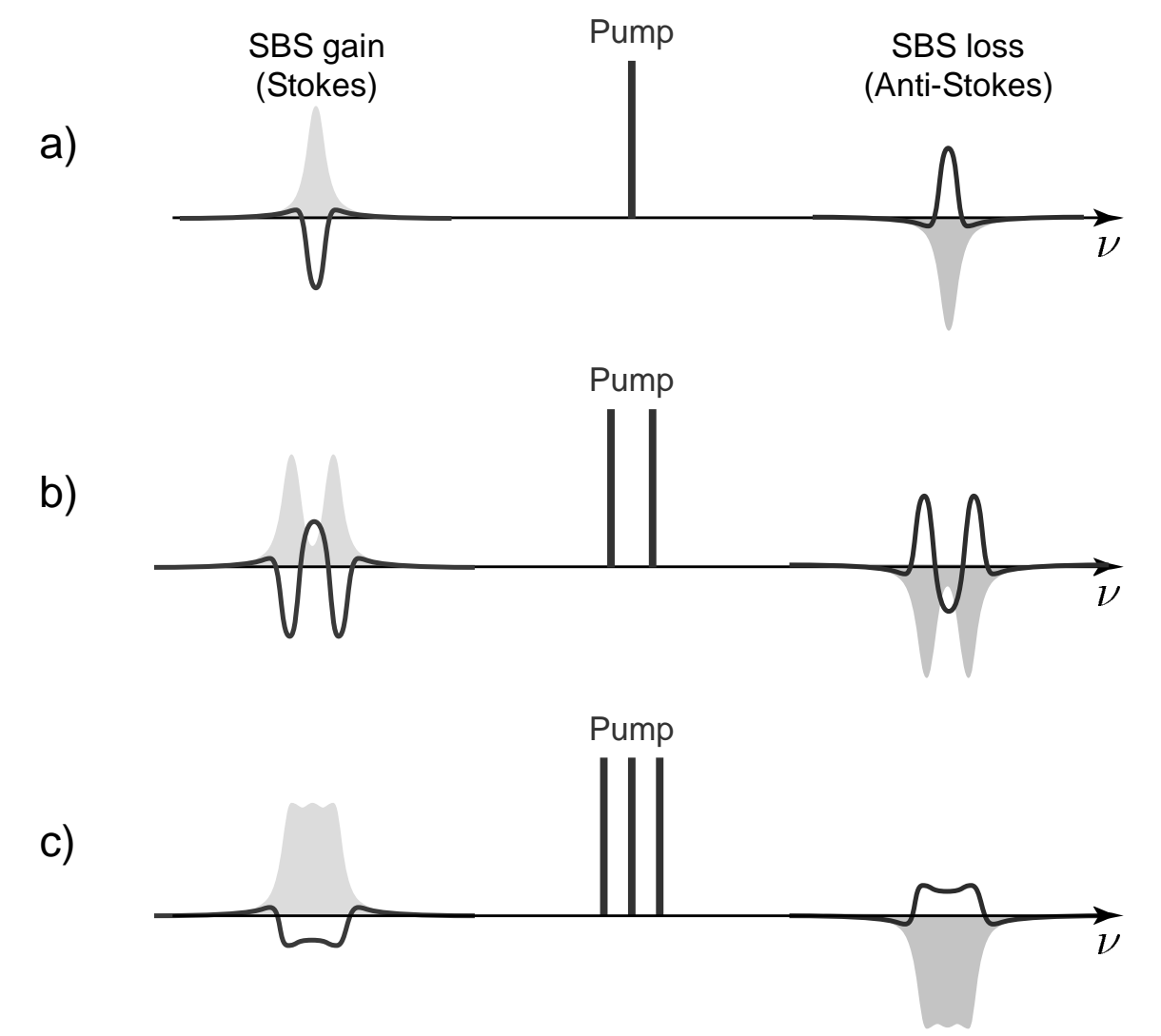

Fig. 2. a) In the SBS process a monochromatic pump gives a gain and a loss Lorentzian spectral distributions (shown in shaded gray), placed symmetrically on both sides and at equal distance by the pump frequency. The group velocity change is given by the solid line and peaks at the center of the gain/loss resonances.

b) In the case of a bichromatic pump the overlapping of the 2 SBS resonances causes an inversion of the curvature at the centre of the spectral distribution that turns into fast light in gain regime (slow light in loss) with a reduced amplitude change.

c) In the case of multi-line pump a relatively flat gain spectrum can be synthesized that results in an enhanced delaying effect and a reduced distortion.

\subsection{Modulation using a continuous broadband spectrum}

Nevertheless, all these experiments that use a moderate number of discrete lines in the pump spectrum do not completely overcome the limitation caused by the narrowband nature of SBS. However, the effective Brillouin gain spectrum can be continuously broadened using a randomly modulated pump source ${ }^{18}$, as depicted in Fig. 3a. A broadened smooth SBS gain spectrum of up to $325 \mathrm{MHz}$ FWHM was generated by directly modulating the current of the pump laser diode using a pseudo-random bit sequence at $38 \mathrm{MBit} / \mathrm{s}$. The width of the pump spectrum is basically controlled by the amplitude and the bit rate of the modulating signal. The bit rate is set high enough to ensure that the pump spectrum seen by the counter propagating pulse is homogeneous when integrated over the entire fiber length ( $6.7 \mathrm{~km}$ or $33 \mu$ s transit time) and that the pump time sequence does not therefore modulate the signal. Pulses as short as $2.7 \mathrm{~ns}$ could be delayed in this 
effective gain spectrum in a process formally identical to a natural resonance, but with a linewidth of the gain resonance that can potentially be arbitrarily extended.

This extension of the bandwidth has definitely removed a bottleneck that was previously considered as impossible to overcome using the SBS approach. However, it must be pointed out that this bandwidth extension requires that the pump power is raised proportionally to the amount of spectral broadening to maintain the same normalized delay, and to the square of the relative spectral broadening to keep the same absolute delay. This is because on one hand the peak effective SBS gain is decreased proportionally to the broadening as a result of the convolution, and on the other hand the slow light delay is inversely proportional to the resonance linewidth.

Detailed studies of the broadband SBS delays have been conducted where the pump spectrum was broadened using an electrical noise source superimposed on the injection current of the pump laser diode ${ }^{19} .75 \mathrm{ps}$ pulses could be delayed, convincingly proving that this type of delay line is compatible with a data rate of up to $10 \mathrm{Gbit} / \mathrm{s}$. The SBS gain spectrum could be broadened up to the point where the Stokes and Anti-Stokes bands start to overlap and to mutually neutralize, as shown in Fig. 3b. This effect prevents any further extension of the gain linewidth and can be seen as the limit to the actual broadening. This corresponds to an equivalent gain bandwidth of $12.6 \mathrm{GHz}$ which can be considered the maximum bandwidth obtained by SBS and a single broadened pump.

\section{Pump}

a)

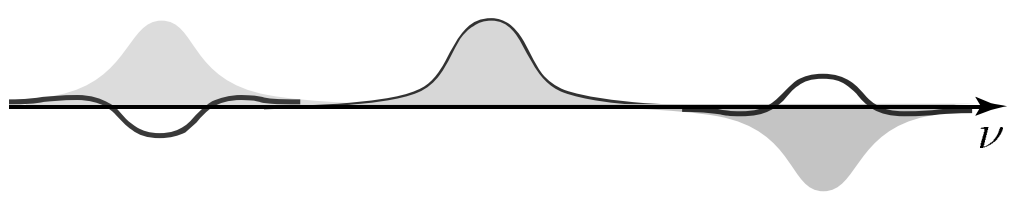

Pump

b)

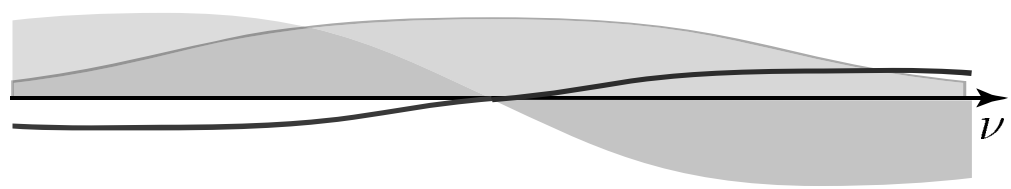

Fig. 3. a) In the case of a homogeneously broadened pump the shape of the gain spectral distribution is a replica of the pump spectrum and broadband delays can be generated at bandwidth far exceeding that of the natural Brillouin resonance.

b) In the extreme case of a broadened pump showing a bandwidth exceeding the Brillouin frequency shift $v_{B}$, the SBS

gain and loss spectra overlap and mutually cancel around the pump frequency, placing a boundary at $v_{B}$ for the maximal gain broadening that can be synthesized.

The effective SBS gain spectra obtained using the pseudo-random bit generator or the noise source provide a typical bellshaped distribution that turns out to be not ideal as far as the phase gradient through the gain resonance and the signal distortion are concerned. An interesting implementation was realized ${ }^{20}$, in which the gain spectral distribution was shaped to maximize the phase gradient and thus the delay strength. The spectrum of a pump semiconductor laser was tailored using a combination of a deterministic, periodic current modulation together with a small random component to obtain a continuous effective SBS gain spectrum with sharp edges. For equal pump powers and gain bandwidths, such a tailored gain spectrum introduces 30-40\% longer delays than a standard Lorentzian resonance. This approach was further developed to optimize both the delay and the distortion and it was found that the ideal gain spectral distribution was approximately square-shaped ${ }^{16,21-23}$.

Extending the bandwidth has a serious cost in terms of pump power. At $10 \mathrm{GHz}$, an equivalent fractional delay to that obtained using the natural non-broadened Brillouin resonance requires a 350 times larger pump power! Considering that the minimal pump power to obtain a fractional delay of 1 (one pulse width) is $5 \mathrm{~mW}$ in very long fibers using the natural resonance, this would bring the required pumping level to $1.75 \mathrm{~W}$ and above to secure a suitable delay at $10 \mathrm{GBit} / \mathrm{s}$. 


\section{ADVANCED DELAY SCHEMES COMBINING SBS GAIN AND LOSS}

Stimulated Brillouin scattering offers an additional degree of freedom through the superposition of gain and loss spectral distributions. This can be realized by using pumps separated by twice the Brillouin frequency shift $v_{B}(\sim 21-22 \mathrm{GHz}$ at $\lambda=1550 \mathrm{~nm}$ ), in which case the Brillouin loss spectrum (Anti-Stokes band) of the lower frequency Pump 1 superposes the Brillouin gain spectrum (Stokes band) of the higher frequency Pump 2, as shown in Fig. 4 and Fig. 5. This possibility offers new functionalities through the complete or partial overlapping of gain and loss spectral distributions with either identical or different broadening.

This feature was exploited to further extend the bandwidth of a SBS delay line over the $12 \mathrm{GHz}$ limit provided by a single pump laser ${ }^{24}$. Two pumps were exactly separated by twice the Brillouin frequency shift $v_{B}$ and identically broadened, so that the loss spectrum of Pump 1 is perfectly cancelled by the gain spectrum of Pump 2 at any frequency. This way the gain spectrum of Pump 1 is no longer compensated by its own loss spectrum and the effective gain broadening can be extended until the loss spectrum of Pump 2 starts to overlap the gain spectrum of Pump 1. The situation is depicted in Fig. 4 and it shows that the limit of the effective gain linewidth can be actually doubled to reach $25 \mathrm{GHz}^{24}$.

This scheme can be further extended by adding another broadened pump separated from pump 2 by the Brillouin shift $v_{B}$ to compensate the loss spectrum of pump 2. This extends the bandwidth by another $12 \mathrm{GHz}$ to get close to the $40 \mathrm{GHz}$ limit needed for ultra-high data rate optical communications. The bandwidth can thus be increased by $v_{B}$ steps by incrementing the number of pumps. However, this raises a serious practical difficulty since such a scheme would require a massive broadening of the pumps emission spectrum, with the consequence that the power of each individual pump must also be increased in the same proportion to maintain the fractional delaying strength.

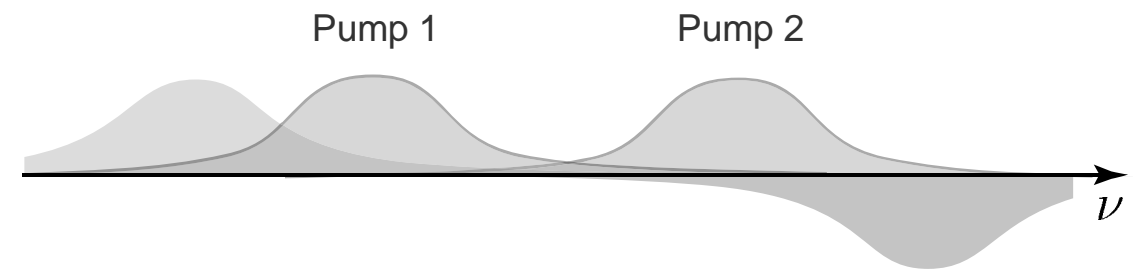

Fig. 4. Two widely broadened pumps with identical bandwidth make their gain and loss spectra exactly compensate in the medium frequency range. The loss of Pump 2 is thus compensated by the gain of Pump 1 and no longer cancels the

gain of Pump 2 that can be further broadened up to twice the Brillouin shift $v_{B}$.

The superposition of gain and loss spectra of different spectral widths also offers the possibility to achieve slow and fast light in a fully transparent regime. A transmission spectrum similar to that obtained by electromagnetically induced transparency can be synthesized, since a narrowband gain can open a transparency window in a broadband loss spectrum. This situation is depicted in Fig. 5a and clearly shows that the gain compensation maintains the sharp spectral transitions necessary to induce slow and fast light propagation. This was experimentally demonstrated ${ }^{25}$ by realizing delays similar to those obtained using a single monochromatic pump, but with less than $1 \mathrm{~dB}$ variations in the signal intensity over an entire $12 \mathrm{~ns}$ delay range. Signal advancement using fast light was also observed by simply swapping the pumps spectral positions. This result has an important practical impact, since any change in the signal intensity remains an unwanted side effect of the delay process. It must be pointed out that the physical nature of the transparency in this case is entirely different to electromagnetically induced transparency and in this gain-loss compensation scheme the signal unfortunately contains all the excess noise resulting from the amplification process.

Following the same approach, gain compensation by a superimposed broadband loss or by two narrowband losses placed on the wings of the gain spectral distribution, as shown in Fig. 5b, offers the possibility to enhance the delaying capability up to twice when compared to a single resonance scheme ${ }^{26}$. 


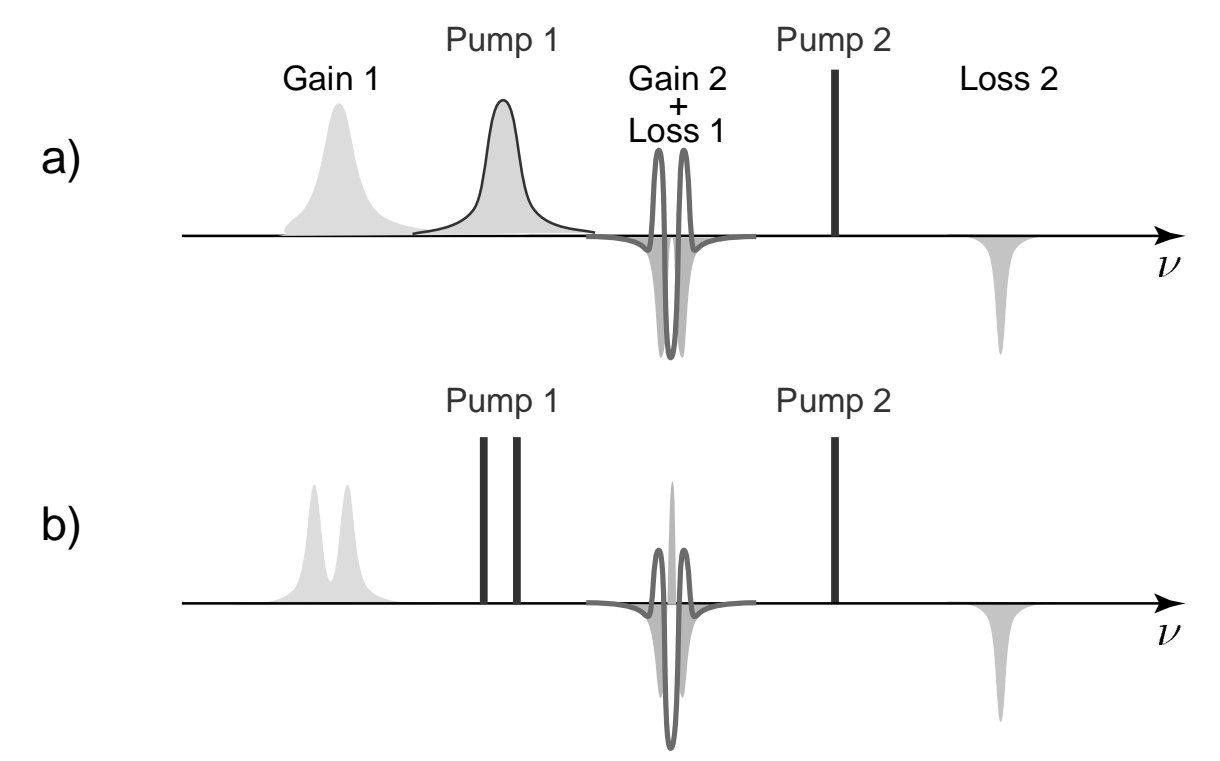

Fig. 5. Advanced delay schemes obtained through the superposition of SBS gain and loss spectra generated by 2 distinct pump separated in frequency by twice the Brillouin shift $v_{B}$. The resulting gain spectral distribution mid-positioned between the two pump is shown in shaded gray and the corresponding group velocity change by a solid line. a) Zero gain delays are obtained by using two pumps with substantially different broadening. The peak gains and losses created by the 2 pumps are identical and cancel at the centre of their overlapping range.

b) Enhanced delaying with the same amplification can be obtained by superposing the spectra of a monochromatic and a bichromatic pump.

Recently, self-generated fast light, in total absence of any pump source, has been reported ${ }^{27}$. In this implementation, the signal is amplified above the critical Brillouin power, often named Brillouin "threshold", so that all the signal light is converted into a backwards propagating wave resulting from amplified spontaneous emission. This intense light is spectrally positionned below the signal frequency by exactly the fiber Brillouin shift and a Brillouin loss resonance exactly positioned at the signal frequency is created. In a $12 \mathrm{~km}$ fiber temporal advancements of up to $12 \mathrm{~ns}$ were continuously generated by varying the signal input power between 10 and $630 \mathrm{~mW}$. This implementation offers many practical advantages in addition to its inherent simplicity, such as a constant signal amplitude at the exit of the delay line and the interesting property that the spectrum of the amplified Brillouin emission matches the signal spectral distribution. In other words it means that the SBS effective loss spectrum self-adapts to the signal spectral distribution and automatically matches its bandwidth.

\section{LIMITS TO BROADENING REDUCTION IN A LINEAR SBS SLOW LIGHT SYSTEM}

The achievement of gain profiles that would yield "zero-broadening" of the pulses travelling through it was claimed and reported in a recent publication ${ }^{10}$. Without surprise the gain profiles suggested in this report are fairly similar to the ones already proposed in preceding works aiming at optimizing the pulse distortion ${ }^{7,9}$. This approach was further developed and experimentally implemented, even showing pulse compression in the slow light medium ${ }^{11}$. The principles for broadening compensation stated in Ref. ${ }^{10}$ are based on the hasty and unjustified assumption that gain and dispersion broadenings add arithmetically to eventually cancel when they show opposite signs. While purely speculative, this statement has misled the later experimental work in Ref. ${ }^{11}$.

We would like to clarify here this issue, and demonstrate the impossibility to compensate amplitude and phase broadenings in any stationary linear system (SLS) such as those proposed in Ref. ${ }^{10} \&{ }^{11}$. At first glance this can be viewed very simply by representing the SLS transfer function by $\exp \{\mathrm{f}(v)\}$ and by considering that the signal amplitude is changed by the real part of $\mathrm{f}(v)$ and the phase by the imaginary part of $\mathrm{f}(v)$. Since a purely real and a purely imaginary quantity cannot mutually cancel, their global effect is scaled by the modulus of $\mathrm{f}(v)$ and is thus given by the geometrical 
sum instead of the simple arithmetical one stated in Ref. ${ }^{10}$. This is illustrated by the repeatedly observed effect that linear chromatic dispersion can never lead to a compressed pulse narrower than the original un-chirped pulse.

Additionally, we demonstrate that all linear slow light systems like the ones proposed in Ref. ${ }^{10} \&{ }^{11}$, that introduce a low-pass filtering of the input spectrum (a reduction in the signal rms spectral width), will necessarily introduce a temporal broadening of a Gaussian un-chirped pulse. This means that such media will never be good candidates for making "zero-broadening" slow light.

In these recent works the width of the output pulses is measured as the full-width at half maximum (FWHM) and is compared with that at the input. It is well-known that the FWHM is not a convenient comparison when the pulses change the shape strongly, as it is the case in this system. The reason is that this measure of pulse width neglects any portion of the signal that is below the half-maximum. With such definition, a very important amount of energy in the pulse lying far from the main peak of the output pulse is simply ignored. This part can lead to a severe intersymbol interference in a real system and is simply not considered in the measure of width that is used.

Additionally, it is literally claimed in Ref. ${ }^{11}$ that "Zero-broadening is possible since the broadening due to the narrow Brillouin gain bandwidth can be compensated by the group velocity dispersion accompanied with the pulse delay". In other words, it is claimed that the amplitude distortion introduced by the low-pass filtering of the slow-light element can be compensated by the group velocity dispersion introduced by it. As sketched at the beginning of this section we show below that this statement is not correct. Moreover the authors were mislead by another erroneous interpretation by Wang et $\mathrm{al}^{10}$ that the phase broadening in the linear slow light system is due to group velocity dispersion like observed during the passive propagation in single mode fibers. For symmetry reasons and as already clearly pointed out in a former work ${ }^{7}$ the phase broadening can only originate from the third-order dispersion, as clarified below.

To develop our proof, we start by defining a generalized metric of pulse width and pulse broadening. The definition that we use of pulse width is the rms temporal width of the pulse:

$$
\sigma_{i}^{2}=\frac{1}{E} \int_{-\infty}^{+\infty} t^{2}|A(t)|^{2} d t
$$

where $A(t)$ is the temporal amplitude of the pulse and

$$
E=\int_{-\infty}^{+\infty}|A(t)|^{2} d t
$$

is the total energy inside the pulse.

For simplicity, we have considered that the pulse is always centered at $t=0$. This simplifies the relations, and the results do not lose any generality. The rms pulse width is the definition of pulse width that is conventionally used in fiber-optic communication systems. This definition of pulse width measures how much the pulse energy is temporally "spread", regardless of the shape.

Let us now consider that the Fourier transform of the pulse envelope reads $A(\omega) \exp [i \varphi(\omega)]$, where $\varphi(\omega)$ has a complicated shape owing to the dispersion introduced by the slow light element, nevertheless showing an odd symmetry. Using the well-known properties of the Fourier transform, we can rewrite the rms pulse width in a way that shows the relative contributions to it by the spectral amplitude $A(\omega)$ and the spectral phase $\varphi(\omega)$ :

$$
\sigma_{i}^{2}=\frac{1}{E}\left[\int_{-\infty}^{+\infty}\left|\frac{d A(\omega)}{d \omega}\right|^{2} d \omega+\int_{-\infty}^{+\infty}|A(\omega)|^{2}\left(\frac{d \phi(\omega)}{d \omega}\right)^{2} d \omega\right]
$$

where, for simplicity, it has been assumed that the mean group delay has been subtracted from $\frac{d \varphi(\omega)}{d \omega}$ (as stated before, the pulse is centred in $t=0$ ). Equation (4) shows that there are two main contributions to the temporal broadening of the pulse: one is due to the variations in the spectral amplitude of the signal, that can be modified through the slow light system by the spectral filtering effect, the so-called gain broadening. The other is due to the variations of the spectral phase in the pulse, and can thus be related to the phase distortion introduced by the medium. It is frequently designated as dispersion broadening in the literature. As it is visible, both contributions are positive, and therefore, they cannot cancel each other. In other words, given a predefined spectral amplitude of the output pulse, the best spectral phase distribution to get the shorter pulse width is the flat one, corresponding to an unchirped pulse. We can equivalently say 
that the group velocity dispersion will never be responsible of a temporal narrowing in the output pulse unless it is prechirped at the input. Incidentally the configuration proposed in Ref. ${ }^{11}$ turns out to be a good illustration of a linear slow light system limited by phase broadening, in which the dominant term is the second term in Equ.(4).

The result given above proves that the phase response of the linear slow light system can never compensate, in terms of broadening, the distortion introduced by the amplitude response. It would nevertheless be interesting to know if there is any possibility of achieving such a zero-broadening slow light system with any kind of gain profile similar to the ones used in Ref. ${ }^{10} \&{ }^{11}$. Although we cannot give a general response to this question, we can easily show that, if a system introduces a low-pass filtering effect in the signal (a reduction in the rms spectral width), any Gaussian pulse at the input will be broadened at the output. To show this, we can define the rms spectral width as:

$$
\sigma_{\omega}^{2}=\frac{1}{2 \pi E} \int_{-\infty}^{+\infty} \omega^{2}|A(\omega)|^{2} d \omega
$$

The well-known uncertainty principle states that as long as $A(t)$ is absolutely continuous and the functions $t E(t)$ and $E^{\prime}(t)$ are square integrable:

$$
\sigma_{i} \sigma_{\omega} \geq \frac{1}{2}
$$

where the equality only holds for a Gaussian pulse, therefore $A(t)=C \exp \left(-t^{2} / \sigma_{0 t}{ }^{2}\right)$. Let us consider this case as the input pulse to our linear slow light medium. The input spectral width is therefore $\sigma_{0 \omega}=1 /\left(2 \sigma_{0 t}\right)$. One common thing to all the slow light systems proposed in Ref. ${ }^{10} \&{ }^{11}$ is that their transfer function $H(\omega)$ is inherently low-pass. Therefore, the output rms spectral width will necessarily be smaller than the input spectral width, thus $\sigma_{\omega}<\sigma_{0 \omega}$. Now, if we consider the uncertainty principle stated in Equ. (6), the output spectral width will be $\sigma_{t} \geq 1 /\left(2 \sigma_{\omega}\right)$. Since $\sigma_{\omega}<\sigma_{0 \omega}$, it follows obviously that $\sigma_{t}>1 /\left(2 \sigma_{0 \omega}\right)=\sigma_{0 t}$. Thus, the output pulse width is always larger than the input pulse. This means that, in general, low-pass linear elements (which have been, so far, all the amplifier-based slow light elements proposed in the literature) will never be candidates to make zero-broadening slow light. There remains an open question on whether any other kind of profile would be able to achieve zero-broadening of pulses. We must remind here, nevertheless, that an extensive, computer-based search for the "optimum" gain profile (that introduces the maximum fractional delay with the minimum distortion in the pulse) was already carried out by Pant et al. ${ }^{9}$, finding that the square-shaped low-pass response was the best possible in SBS-based slow light systems.

We would like also to stress that ordinary group velocity dispersion as observed in single mode fibers can in no way lead to a good compensation of phase broadening in a slow light linear system. For a pulse placed at the center of the gain spectrum and for symmetry reasons the amplitude part of the transfer function $A(\omega)$ will show a symmetric distribution around the central frequency $\omega_{\mathrm{o}}$ while the phase part $\varphi(\omega)$ will be anti-symmetric and thus only contains odd terms in its polynomial expansion. The first term in this odd expansion gives the group delaying effect and the following term, responsible for distortion and phase broadening, is the third-order dispersion that depends cubically on the frequency. It can thus never be cancelled by a quadratic term as induced by ordinary group velocity dispersion, as suggested in Ref. ${ }^{10}$ $\&^{11}$.

In summary, we have shown that phase distortion can never lead to the compensation of the low pass filtering effect in slow light systems. Additionally, we have shown that all the linear slow light systems showing a low-pass response (such as all the amplifier-based ones) will never be candidates for making zero-broadening slow light. We should stress that this impossibility is limited to linear systems and it might be possible, however, to compensate the broadening after the slow light system by inserting suitable nonlinear elements (such as, for instance, saturable absorbers). The role of these nonlinear elements would be, eventually, to "re-generate" the frequencies that have been filtered out by the slow light element. 


\section{CONCLUSIONS}

Slow- and fast-light delay lines in optical fibres based on SBS shows unprecedented flexibility such as the ability to shape the spectral resonance possible through the modulation of the pump. In recent years, attractive solutions have been proposed to overcome the challenges associated with use in real applications. These solutions include the realization of broadband delays up to $25 \mathrm{GHz}$ and more, optimization of the gain spectral distribution for signal delay with minimal distortion, the achievement of delays in metre-long fibres and the realization of delays with no amplitude change.

The research in this field is now clearly at a turning point and the next step requires the optimization of the actual systems through the maximisation of the delay while minimizing the distortion experienced by the signal. In this view the possibility to fully engineer the gain spectrum is clearly a key advantage uniquely offered by stimulated Brillouin scattering in optical fibers. Some solutions have been recently proposed based on linear systems to cancel the pulse broadening inherent to the low-pass transfer function of the delaying process, but we have shown that so far this remains essentially a delusion. Certainly other clever solutions will be soon proposed to make slow \& fast light in optical fibers a real timing tool for photonics.

\section{ACKNOWLEDGEMENTS}

We acknowledge the support from the Swiss National Science Foundation through project 200021-109773, the Spanish Ministry of Education and Science through project TEC2006-09990-C02-02 and the European Community's Seventh Framework Programme [FP7/2007-2013] under grant agreement nº 219299 (GOSPEL project).

\section{REFERENCES}

[1] L. Thevenaz, "Slow and fast light in optical fibres," Nat Photon 2(8), 474-481 (2008)

[2] K. Y. Song, M. Gonzalez Herraez and L. Thevenaz, "Observation of pulse delaying and advancement in optical fibers using stimulated Brillouin scattering," Optics Express 13(1), 82-88 (2005)

[3] Y. Okawachi, M. S. Bigelow, J. E. Sharping, Z. M. Zhu, A. Schweinsberg, D. J. Gauthier, R. W. Boyd and A. L. Gaeta, "Tunable all-optical delays via Brillouin slow light in an optical fiber," Physical Review Letters 94(15), $153902(2005)$

[4] K. Y. Song, M. G. Herraez and L. Thevenaz, "Long optically controlled delays in optical fibers," Optics Letters 30(14), 1782-1784 (2005)

[5] M. Gonzalez-Herraez, K. Y. Song and L. Thevenaz, "Optically controlled slow and fast light in optical fibers using stimulated Brillouin scattering," Applied Physics Letters 87(8), - (2005)

[6] M. D. Stenner, M. A. Neifeld, Z. Zhu, A. M. C. Dawes and D. J. Gauthier, "Distortion management in slow-light pulse delay," Opt. Express 13(25), 9995-10002 (2005)

[7] J. B. Khurgin, "Performance limits of delay lines based on optical amplifiers," Optics Letters 31(7), 948-950 (2006)

[8] A. Zadok, A. Eyal and M. Tur, "Extended delay of broadband signals in stimulated Brillouin scattering slow light using synthesized pump chirp," Optics Express 14(19), 8498-8505 (2006)

[9] R. Pant, M. D. Stenner, M. A. Neifeld and D. J. Gauthier, "Optimal pump profile designs for broadband SBS slowlight systems," Optics Express 16(4), 2764-2777 (2008)

[10] S. Wang, L. Ren, Y. Liu and Y. Tomita, "Zero-broadening SBS slow light propagation in an optical fiber using two broadband pump beams," Opt. Express 16(11), 8067-8076 (2008)

[11] T. Schneider, A. Wiatrek and R. Henker, "Zero-broadening and pulse compression slow light in an optical fiber at high pulse delays," Opt. Express 16(20), 15617-15622 (2008)

[12] K. Y. Song, M. Gonzalez Herraez and L. Thevenaz, "Gain-assisted pulse advancement using single and double Brillouin gain peaks in optical fibers," Opt. Express 13(24), 9758-9765 (2005)

[13] Z. M. Zhu and D. J. Gauthier, "Nearly transparent SBS slow light in an optical fiber," Opt. Express 14(16), 72387245 (2006)

[14] A. Minardo, R. Bernini and L. Zeni, "Low distortion Brillouin slow light in optical fibers using AM modulation," Opt. Express 14(13), 5866-5876 (2006) 
[15] Z. Shi, R. Pant, Z. Zhu, M. D. Stenner, M. A. Neifeld, D. J. Gauthier and R. W. Boyd, "Design of a tunable timedelay element using multiple gain lines for increased fractional delay with high data fidelity," Opt. Lett. 32(14), 1986-1988 (2007)

[16] Z. Lu, Y. Dong and Q. Li, "Slow light in multi-line Brillouin gain spectrum," Opt. Express 15(4), 1871-1877 (2007)

[17] Y. Dong, Z. Lu, Q. Li and Y. Liu, "Broadband Brillouin slow light based on multifrequency phase modulation in optical fibers," J. Opt. Soc. Am. B 25(12), C109-C115 (2008)

[18] M. Gonzalez Herraez, K. Y. Song and L. Thevenaz, "Arbitrary-bandwidth Brillouin slow light in optical fibers," Opt. Express 14(4), 1395-1400 (2006)

[19] Z. M. Zhu, A. M. C. Dawes, D. J. Gauthier, L. Zhang and A. E. Willner, "Broadband SBS slow light in an optical fiber," J. Lightwave Technol. 25(1), 201-206 (2007)

[20] A. Zadok, A. Eyal and M. Tur, "Extended delay of broadband signals in stimulated Brillouin scattering slow light using synthesized pump chirp," Opt. Express 14(19), 8498-8505 (2006)

[21] J. B. Khurgin, "Performance limits of delay lines based on optical amplifiers," Opt. Lett. 31(7), 948-950 (2006)

[22] R. Pant, M. D. Stenner, M. A. Neifeld and D. J. Gauthier, "Optimal pump profile designs for broadband SBS slowlight systems," Opt. Express 16(4), 2764-2777 (2008)

[23] L. Yi, Y. Jaouen, W. Hu, Y. Su and S. Bigo, "Improved slow-light performance of 10 Gb/sNRZ, PSBT and DPSK signals in fiberbroadband SBS," Opt. Express 15(25), 16972-16979 (2007)

[24] K. Y. Song and K. Hotate, "25 GHz bandwidth Brillouin slow light in optical fibers," Opt. Lett. 32(3), 217-219 (2007)

[25] S. Chin, M. Gonzalez Herraez and L. Thevenaz, "Zero-gain slow \& fast light propagation in an optical fiber," Opt. Express 14(22), 10684-10692 (2006)

[26] T. Schneider, R. Henker, K. U. Lauterbach and M. Junker, "Comparison of delay enhancement mechanisms for SBS-based slow light systems," Opt. Express 15(15), 9606-9613 (2007)

[27] S. Chin, M. Gonzalez-Herraez and L. Thevenaz, "Self-advanced fast light propagation in an optical fiber based on Brillouin scattering," Opt. Express 16(16), 12181-12189 (2008) 